\title{
Resource competition and species coexistence in a two-patch metaecosystem model
}

\author{
loannis Tsakalakis ${ }^{1} \cdot$ Bernd Blasius $^{1,2} \cdot$ Alexey Ryabov $^{1}$ (D) \\ Received: 24 April 2019 / Accepted: 20 September 2019 \\ (C) Springer Nature B.V. 2019
}

\begin{abstract}
The metaecosystem framework has been proposed to conceptualize the interactive effects of dispersal and resource flows on the structure and functioning of communities in a heterogeneous environment. Here, we model a two-patch metaecosystem where two species with a trade-off in resource requirements compete for two limiting resources - generalizing the so-called gradostat experimental setup. We study the competition outcome in dependence of resource heterogeneity and between-patch diffusion for different combinations of resource supply ratios. Our numerical simulations show that community composition and local and regional diversity are determined by the interplay of resource heterogeneity, resource supply stoichiometry, and diffusion rate. High resource heterogeneity increases regional diversity, with species coexisting due to spatial segregation, whereas low resource heterogeneity favors local diversity, as species coexist mainly by local resource partitioning. Regional diversity averaged across a gradient of resource ratios decreases monotonically with diffusion rate, while local diversity follows a unimodal dependency. However, these dependencies become bimodal for high resource heterogeneity because various bistable states occur at intermediate diffusion rates. We identify three kinds of bistable states with species priority effect: (i) bistability between the dominance of one or the other competitor, (ii) bistability between one species dominance or species coexistence, and (iii) two alternative coexistence regimes differing in species-relative abundances. Most bistable states appear at high resource levels when biomass fluxes strongly interact with resource fluxes. Our analysis provides new insights for the potential effects of metaecosystem dynamics on biodiversity patterns.
\end{abstract}

Keywords Metaecosystem · Resource competition · Bistability · Dispersal · Spatial heterogeneity · Species coexistence · Biodiversity

\section{Introduction}

The role of connectivity for ecosystem functioning has received much attention in modern ecology. The inflows and outflows of organisms and resources can significantly affect and be affected by the ecosystem structure, functioning, and

Electronic supplementary material The online version of this article (https://doi.org/10.1007/s12080-019-00442-w) contains supplementary material, which is available to authorized users.

Alexey Ryabov

alexey.ryabov@uni-oldenburg.de

1 Institute for Chemistry and Biology of the Marine Environment (ICBM), University of Oldenburg, Oldenburg, Germany

2 Helmholtz-Institute for Functional Marine Biodiversity at the University Oldenburg (HIFMB), University of Oldenburg, Oldenburg, Germany internal resource level. The concept of a meta-community was proposed to shed light on the effects of dispersal between adjacent patches of an ecosystem (Wilson 1992; Leibold et al. 2004). In the meta-community concept, a community is no longer viewed as an isolated system but as a part of a regional network of local communities interacting with each other through species dispersal. Source-sink dynamics is a major process constraining meta-communities, considering that one competitor or a group of organisms are better adapted in a given patch of the ecosystem (source); however, connectivity allows the same organisms to be present in adjacent patches (sink) (Mouquet and Loreau 2003). To examine the effects of both dispersal of organisms and resource flows, the meta-community concept was later extended to that of a metaecosystem (Loreau et al. 2003b). Both concepts have been proven to be important tools to link environmental connectivity with biodiversity (Mouquet and Loreau 2003; Gravel et al. 2010b), productivity (Mouquet et al. 2002; 
Loreau et al. 2003a; Hodapp et al. 2016), and ecosystem stability (Gounand et al. 2014; Gravel et al. 2016).

The existence of resource gradients is a major factor affecting community structure in heterogeneous environments. Under homogeneous conditions, resource-ratio theory links the possibility of species coexistence with trade-offs in the species' competitive traits (Tilman 1980, 1982). Given only one limiting resource, the species with the highest competitive ability for that resource (i.e., the species with the lowest $\mathrm{R}^{*}$ value) will outcompete all other species. When species compete for more than one limiting resource, they can coexist if they have a trade-off in their $R^{*}$-values for these resources. Resource-ratio theory was later extended from homogeneous to heterogeneous environments, supporting the idea that a spatial gradient of different resource ratios should favor the dominance of different species at different localities, maintaining, therefore, a highly diverse community on a regional scale (Tilman 1985). However, studies of resource-ratio theory have typically considered only local competition, while metacommunity and metaecosystem theories have shown that species dispersal and resource flows can significantly alter competitive interactions (Abrams and Wilson 2004; Gravel et al. 2010a; Ryabov and Blasius 2011, 2014).

A simple experimental setup that fits the definition of a metaecosystem, the so-called gradostat, was suggested a long time ago by Lovitt and Wimpenny (1981) to examine microbial community structure in a spatial system with resource gradients. A gradostat represents a chain of patches in which neighboring patches are interconnected by diffusion of species and resources. Two limiting resources are supplied at the opposite ends of the chain, generating resource heterogeneity and creating different spatial niches for the competitors. Experimental studies have shown that increasing spatial resource heterogeneity in gradostats also increases local diversity (Codeço et al. 2001) and dissimilarity in species composition among the patches (Gülzow et al. 2018). Resource gradients can affect the distribution of species functional traits and community structure (Gounand et al. 2017) and increase diversity of phytoplankton and bacteria (Limberger et al. 2017).

The notion of a metaecosystem underpins conceptualization of the effects of species traits and diffusion (of both species and resources) on community composition and functioning (Gross and Cardinale 2007; Gravel et al. 2010a; Marleau et al. 2015; Hodapp et al. 2016). It has been shown that heterogeneous distributions of resources can lead to alternative stable states (Ryabov et al. 2010; Ryabov and Blasius 2014) and that species traits that favor coexistence in a homogeneous system may lead to alternative stables states in a heterogeneous environments, and vice versa (Ryabov and Blasius 2011). Following the seminal work by Tilman (1980), a number of graphical approaches have been suggested to analyze the outcome of competition in a patchy environment for one resource (Haegeman and Loreau 2015), and for two resources in a uniformly and weakly mixed water column (Huisman and Weissing 1994; Ryabov and Blasius 2011). However, the effects of system connectivity and species traits on the competition outcome and diversity in a patchy environment, where the supplies of two resources are heterogeneously distributed, are still not clear.

In this study, we use a two-patch metaecosystem model, where two species share two limiting resources. The two patches are connected by diffusion of both organisms and abiotic resources. Using this set up, we vary the spatial distribution of resource supplies from a homogeneous distribution where both patches are supplied with exactly the same amount of both resources, to the maximally heterogeneous case (gradostat) where each patch is directly supplied with only one of the two resources (while the other resource can only diffuse from the opposite patch). Our approach generalizes the original gradostat setup (Lovitt and Wimpenny 1981) in such a way that by changing the heterogeneity of resource distribution in the model, we keep the total resource load in the metaecosystem constant. This approach enables us to better compare the results obtained for various levels of resource heterogeneity. Using our model setup, we study the effects of diffusion rate and resource heterogeneity on species composition, local and regional biodiversity, and biomass for various ratios of resource supplies, making predictions on how resource heterogeneity and diffusion can affect species coexistence and biodiversity in metaecosystems.

\section{Methods}

We model competition between two species for two limiting resources in a metaecosystem consisting of two diffusively connected patches, where resource 1 and resource 2 are supplied with different rates into each patch. This heterogeneity in resource supplies causes fluxes of resources and species biomass between the patches (Fig. 1). We assume that both competitors have the same maximal growth rate and a trade-off in the minimal requirements for limiting resources (so-called $R^{*}$ values): species 1 is a better competitor for resource 1 and requires relatively more of the resource 2 , and vice versa for species 2 (see species ZNGIs in Fig. S1). The consumption coefficients (a unit of resource consumed to produce a unit of biomass) are chosen in such a way that the two competitors can coexist in a homogeneous environment, e.g., each species consumes relatively less that resource for which it is the best competitor (Tilman 1980).

Species growth rates in each patch follow the classic Monod kinetics of resource availability and Liebig's law of the minimum (León and Tumpson 1975; Grover 1997): 


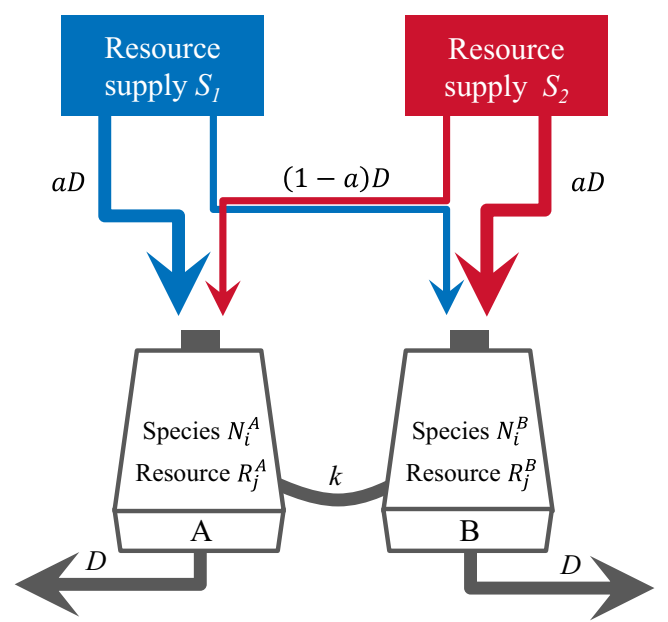

Fig. 1 Generalized gradostat model consisting of two diffusively connected patches (A and B) supplied with the essential resources $R_{I}$ (blue) and $R_{2}$ (red) with dilution rate $D$. Two species of biomass $N_{i}$ with a trade-off in resource requirements compete for these resources. The two patches are coupled by diffusion of biomass and of resources with strength $k$. The heterogeneity of resource supply distribution is controlled by the fraction $a(0.5<\mathrm{a}<1)$ of resource 1 supplied into one patch $\mathrm{A}$ and of resource 2 supplied into patch B. Homogeneous resource distribution corresponds to $a=0.5$ where each patch receives the same amount of resources; maximally heterogeneous resource distribution (gradostatlike scenario) corresponds to $a=1$, where patch A receives only resource 1 and patch $\mathrm{B}$ only resource 2

$\mu_{i}^{X}=\mu_{\max } \min \left\{\frac{R_{1}^{X}}{K_{i 1}+R_{1}^{X}}, \frac{R_{2}^{X}}{K_{i 2}+R_{2}^{X}}\right\}$.

Here, $\mu_{i}^{X}$ is the growth rate of species $i$ in patch $X(X=$ $A$ or $B), \mu_{\max }$ is the maximum growth rate, $R_{j}^{X}$ the concentration of resource $j$ in patch $X$, and $K_{i j}$ the half-saturation constant of species $i$ for resource $j$. Note that we use dimensionless variables for species growth parameters and resource concentrations (Table 1). We normalize units for each of the two resources by taking the half-saturation constant of the weakest competitor for the respective resource to be one.
The minimal resource requirement $R_{i j}^{*}$ of species $i$ for resource $j$ is defined as the resource concentration at which the growth rate equals the dilution rate in a homogeneous system. We denote the dilution rate as $D$ and assume that it is the same in both patches. From the condition $\mu_{i}^{X}\left(R_{1}, R_{2}\right)=D$, we obtain the minimal resource requirements as $R_{i j}^{*}=K_{i j} D /\left(\mu_{\max }-D\right)$. Since the maximal growth and dilution rates are the same for both species, the trade-off in resource requirements translates into a trade-off in half-saturation constants. Note that $R_{i j}^{*}$ values can be calculated using the above simple equation only for a homogeneous system. To estimate the effective minimal resource requirements of competitors in a heterogeneous system, we calculate the minimal resource supply concentrations along the 1:1 diagonal in the resource plane at which at least one of the species survives.

We assume that both biomass and resources can diffuse between patches with the same diffusion rate $k$. Let $N_{i}^{X}$ denote the biomass of species $i$ in patch $X$. Its dynamics follow the equation:

$\frac{d N_{i}^{X}}{d t}=\left(\mu_{i}^{X}-D\right) N_{i}^{X}+k\left(N_{i}^{Y}-N_{i}^{X}\right)$.

Here, the first term describes the local growth and loss of biomass and the second term expresses the diffusion of species $i$ between the patch $X$ and $Y$ (if $X=A$ then $Y=B$ and vice versa). The dynamics of the resource concentration $R_{j}^{X}$ follow the equation:

$\frac{d R_{j}^{X}}{d t}=D\left(S_{j}^{X}-R_{i}^{X}\right)+k\left(R_{j}^{Y}-R_{j}^{X}\right)-\sum_{i} c_{i, j} \mu_{i}^{X} N_{i}^{X}$

where the first term describes the balance between dilution and local supply of the resource with concentration $S_{j}^{X}$, the second term expresses diffusion between the patches, and the last term the consumption of resource $j$ by species $i$, where $c_{i, j}$ are consumption coefficients representing the amount of

Table 1 Species parameters

\begin{tabular}{llll}
\hline Parameter & Symbol & Species 1 & Species 2 \\
\hline Maximal growth rate & $\mu_{\max }$ & 0.7 & 0.7 \\
Half-saturation constant for $R_{l}$ & $K_{i, 1}$ & 0.5 & 1 \\
Half-saturation constant for $R_{2}$ & $K_{i, 2}$ & 1 & 0.5 \\
Consumption coef. for $R_{I}$ & $c_{i, 1}$ & 1.2 & 0.5 \\
Consumption coef. for $R_{2}$ & $c_{i, 2}$ & 0.5 & 1.2 \\
Minimal requirements for $R_{1}$ & $R_{i, 1}^{*}$ & 0.375 & 0.75 \\
Minimal requirements for $R_{2}$ & $R_{i, 2}^{*}$ & 0.75 & 0.375 \\
Dilution rate & $D$ & 0.3 & $0-2.56$ \\
Diffusion rate between patches & $k$ & $0.5-1$ \\
Resource heterogeneity & $a$ &
\end{tabular}


resource $j$ needed to produce a unit biomass of species $i$.

Our model is an extension of the gradostat model suggested by Lovitt and Wimpenny (1981)), who assumed that resource 1 is supplied with rate $D \cdot S_{1}$ into patch $\mathrm{A}$ and diffuses into $\mathrm{B}$, and resource 2 is supplied with rate $D \cdot S_{2}$ into patch $\mathrm{B}$ and diffuses into A. Leading to sharp opposing resource gradients, this model setup does not allow to regulate the level of resource heterogeneity and compare the system functioning for homogeneous and heterogeneous resource distributions. Here, we generalize this approach and suggest a setup for modeling the system ranging from a strongly heterogeneous to a uniform distribution of resources. Let parameter $a$ determine the level of system heterogeneity. Assume that resource 1 is supplied with rate $a D S_{1}$ into patch $\mathrm{A}$ and with rate $(1-a) D S_{1}$ into patch $\mathrm{B}$, while resource 2 with rate $(1-a) D S_{2}$ into patch $\mathrm{A}$ and rate $a D S_{2}$ into B (Fig. 1). When $a=0.5$, each patch obtains $50 \%$ of each resource and the system becomes homogeneous; when $a=1$, then $100 \%$ of $R_{1}$ is supplied into patch $\mathrm{A}$ and $100 \%$ of $R_{2}$ into patch $\mathrm{B}$, so that the resource distribution becomes, like in a gradostat, strongly heterogeneous. Thus, changing $a$ from 0.5 to 1 alters the level of system heterogeneity between these extremes but retains the same global amount of resources supplied into the system, which makes it possible to compare different model outcomes on the same scale.

An increase of $a$ increases the asymmetry in resource distribution between the patches and creates a potential for species coexistence due to spatial segregation. While the global resource supply ratio equals $S_{1} / S_{2}$, the local ratio is $a S_{1} /(1-$ a) $S_{2}$ in patch A and $(1-a) S_{1} / a S_{2}$ in patch B. If, for instance, $a$ approaches 1 then, independently of $S_{1} / S_{2}$, patch A obtains relatively more of resource 1 and patch $B$ relatively more of resource 2 , and differently adapted species obtain their niches in different patches. Below, we call a species "adapted" (or "maladapted") if the ratio in resource requirements of the species match (or do not match) the ratio of total resource supply in the system. Species 1 is adapted to large $S_{1} / S_{2}$, and species 2 to small $S_{1} / S_{2}$. In the homogeneous case, a disbalance in resource supplies always favors the adapted species, and we obtain, for instance, a monoculture of species 1 , when resource 1 is supplied at much higher concentrations compared to resource 2. Under heterogeneous conditions, however, due to a strong asymmetry in resource distribution, a maladapted species can also dominate at such resource supply ratios that would favor the adapted species in a homogeneous system. Below, we refer the dominance of an adapted species as A-monoculture and the dominance of a maladapted species as M-monoculture.

We study how species composition depends on the four main control parameters: the level of resource heterogeneity $a$, the diffusion rate $k$, and the resource supply concentrations $S_{1}$ and $S_{2}$. We simulate the model for a grid of $152 \times 152$ resource supply points. To illustrate the outcome of competition for different levels of heterogeneity on the same scale, we characterize the resource conditions by the average resource concentrations supplied into each patch, $\widetilde{S}_{i}=\left(a S_{i}+(1-a) S_{i}\right) / 2=S_{i} / 2$. Values $\widetilde{S}_{i}$ are more convenient than $S_{i}$, because for homogeneous settings, $\widetilde{S}_{i}$ equal the local resource supplies in each patch, and the results of resource ratio theory can be directly transferred to our twopatch system.

To explore the dependence of competition outcomes on the initial conditions, we apply the invasion criterion (Chesson 2000). Thereby, for each parameter combination, we run two simulations, where either species 1 is the resident and species 2 invades, or vice versa. To obtain a stable solution for the resident species, we run the system for 500 modeling days, then the invader starts to grow from a small initial biomass $\left(N_{i n v, 0}=0.1\right)$ and we run the system for the following 1500 modeling days to find a final solution. This time interval was enough to obtain stable solutions for all parameter combinations.

Biodiversity is estimated as an effective species number $(E S N)$, calculated as the exponential Shannon index (Leinster and Cobbold 2012):

$E S N=e^{-\sum_{i} p_{i} \ln p_{i}}$

where $p_{i}$ is the relative biomass of species $i$. We calculate regional diversity as the $E S N$ in the entire system, using the relative values of the total biomass of each species. Local diversity is calculated as the averaged ESN between the two patches, where $E S N$ is first calculated within each patch, and then the average is taken between the ESNS of the two patches. To separate the effects of diffusion rate and resource heterogeneity from the effects of resource supply ratio, we also analyzed the aggregated biodiversity averaged over a gradient of resource supply ratios shown as a black dashed line in Fig. 2 a.

\section{Results}

The homogeneous model setup (the same concentrations of resources $\left(0.5 S_{1}, 0.5 S_{2}\right)$ are supplied into each patch) reproduces the competition pattern obtained in well-mixed systems (Tilman 1980). In this scenario, species coexist locally at balanced resource ratios, one or another competitor wins at imbalanced resource ratios, and one or both competitors go extinct when the concentration of one of the resources is below the respective species $R^{*}$-value (Fig. 2a). As resource conditions are the same in both patches, the regional pattern of competition outcomes (Fig. 2a) coincides with the local patterns obtained for each patch (Fig. 2c, d) and is independent of the diffusion between the patches.

The regional pattern of competition outcomes drastically changes in a strongly heterogeneous scenario. To first simplify 
Homogeneous two-patch system

a

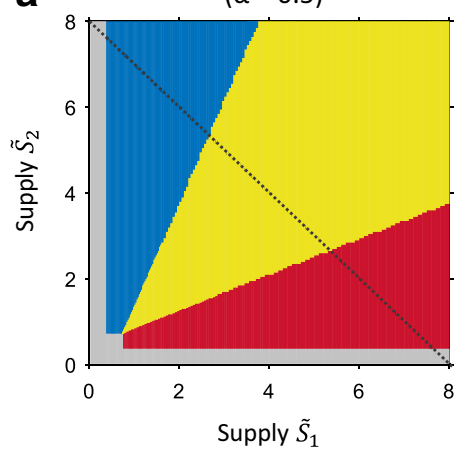

C

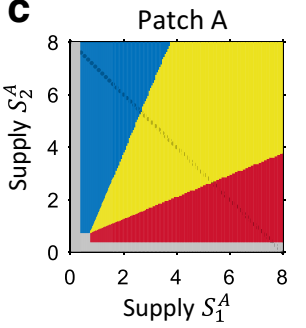

no growth 1st wins

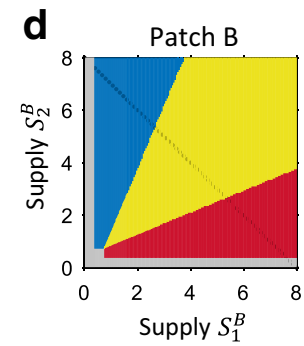

$(\alpha=0.5)$

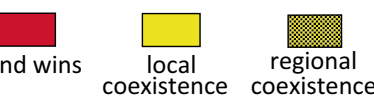

Fig. 2 Partitioning of the regional competition outcomes for the entire system into local competition outcomes in the model without diffusion $(k=0)$. Competition outcomes for homogeneous resource distribution (left panels, $a=0.5$ ) and strongly heterogeneous resource distribution (right panel, $a=0.9$ ). Top panels show the regional competition outcome calculated for the entire system as a function of average resource supplies, $\mathfrak{S}_{j}$, and bottom panels show the local species composition in each patch as a function of local resource supplies, $S_{j}^{X}$

the problem, we consider $a=0.9$ and no diffusion between the patches. Thus, the local resource supplies equal $\left(0.9 S_{1}, 0.1 S_{2}\right)$ in patch $\mathrm{A}$ and $\left(0.1 S_{1}, 0.9 S_{2}\right)$ in patch $\mathrm{B}$, so that each patch obtains $90 \%$ of one resource and only $10 \%$ of the other. Calculating competition outcomes for this scenario (Fig. 2b), we now find in the resource plane four areas where one of the competitors wins (red and blue shading) separated by three areas of coexistence (yellow shading). The areas where both species become extinct (gray) increase in size compared to the homogeneous scenario, indicating an increase in specieseffective minimal resource requirements under heterogeneous conditions.

To understand the regional competition pattern in detail, consider the local patterns obtained for each patch (Fig. 2e, f). Because of the asymmetry in resource distribution, the local patterns in patch A and B look different. However, as the diffusion between the patches equals zero, the local competition outcomes should follow the same rules as in the homogeneous scenario (Fig. 2c, d). To plot these patterns on the same scale as the regional pattern, we add the average resource supplies $\widetilde{S}_{1}$ and $\widetilde{S}_{2}$ as a second scale in the Fig. 2 e, f. The average values $\widetilde{S}_{i}$

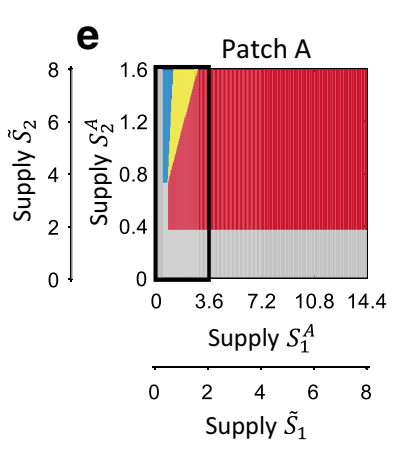

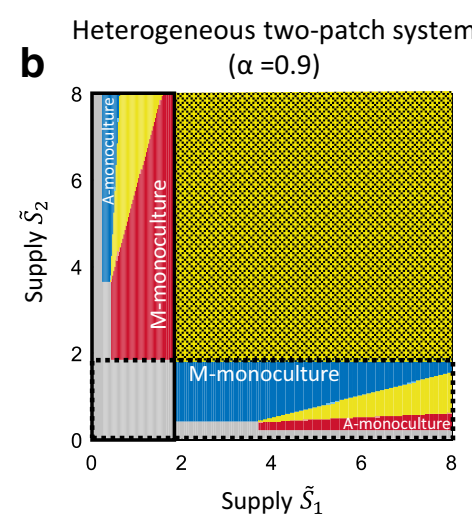

and (for $\mathbf{e}$ and $\mathbf{f}$ only) also as a function of the total average resource supplies, $\mathfrak{S}_{j}$, shown as the second axis. Different competition outcomes are color coded (see legend). The black dotted line in a shows the resource ratios used to calculate changes in the relative biomass of species 2 (Fig. 4) and to find average regional and local diversity (Fig. 6 ) along this gradient of resource ratios

and local values $S_{i}^{X}$ are linearly related. For instance, for patch A, $S_{1}^{A}=2 a \widetilde{S}_{1}$ and $S_{2}^{A}=2(1-a) \widetilde{S}_{2}$. Thus, for $a=0.9$, the change of scale results in stretching the pattern obtained for a homogeneous scenario by the factor $2 a=1.8$ along the vertical axis and squeezing it by a factor $2(1-a)=0.2$ along the horizontal axis (comp. Fig. 2e, c), and the opposite transformation applies to the patch B (comp. Fig. 2f, d).

This transformation affects the location of the areas of various competition outcomes in the resource plane. The local coexistence in each patch is now possible in a much narrower range of resource supplies compared to the homogeneous system (yellow shading). By contrast, the asymmetry in resource distributions provides a broad niche for M-monocultures, where a maladapted species for the given resource supply ratio wins the competition. Species 2 wins for a wide range of parameters in patch A (red shading, Fig. 2e), and species 2 mostly wins in patch B (blue shading, Fig. 2e). The asymmetry of the resource distribution can be compensated, however, by an extremely imbalanced ratio of resource supplies $\left(\widetilde{S}_{1} \gg \widetilde{S}_{2}\right.$ or $\left.\widetilde{S}_{1} \ll \widetilde{S}_{2}\right)$ leading to the appearance of an A-monoculture, when an adapted species wins the competition (blue area in 
Fig. 2e and red in Fig. 2f). The effective minimal resource requirements for the total resource supply (the size of the gray area) also now depend on the patch. Survival in patch A requires a higher average level of resource 2 and smaller average level of resource 1 compared to the homogeneous scenario.

The patterns of local competition outcomes (Fig. 2e, f) help us to classify the regional competition outcomes (Fig. 2b). The middle area of coexistence (yellow area with black hatching) corresponds to regional coexistence, which occurs due to spatial segregation of the competitors, with species 2 dominating in patch $\mathrm{A}$ and species 1 dominating in patch $\mathrm{B}$. By contrast, the other two areas of coexistence (yellow areas without hatching) occur due to local resource partitioning in each patch (as in a homogeneous system) when the asymmetry of resource distribution transforms an unbalanced global resource supply ratio into a balanced local resource ratio. This occurs in patch A (which obtains only $10 \%$ of resource 2 ) when resource 2 is supplied at a relatively higher rate than resource 1 , and in patch $\mathrm{B}$ when resource 1 is supplied at a relatively higher rate than resource 2 .

The ranges of resource supplies at which one species outcompetes the other (blue and red shading in Fig. 2b) also have a more complex structure compared to the homogeneous setup (Fig. 2a). The two largest monoculture areas, at the sides of the regional coexistence area, correspond to M-monocultures, when a maladapted species with respect to the input resource ratio wins in one of the patches, but both species go extinct in the other. In contrast, the two narrower monoculture areas at extreme resource ratios are A-monocultures, corresponding to the dominance of the better adapted species. Extending the boundaries of the resource plane to higher resource supplies leads to an intersection of M-monoculture areas with regional coexistence areas (Fig. S2). As we show below, the interactions between the M-monoculture and local coexistence regimes play an important role when the patches are diffusively coupled.

In the presence of diffusion, flows of species biomass and resources generate source-sink dynamics with the effect to homogenize the resource distribution. Consider the effects of a gradual increase of diffusion on species composition, assuming the same level of heterogeneity $a=0.9$ as before (Fig. 3). For the sake of completeness, this set of figures starts from the setup with no diffusion (Fig. 3a matches Fig. 2b) and ends at a diffusion level that is high enough to homogenize the system, so Fig. 3h approaches Fig. 2a. Studying the transition in competition outcomes between these two extremes allows us to illustrate the effects of diffusion on species composition.

A low diffusion rate (Fig. 3b) leads to only small transformations of the pattern observed with zero diffusion (Fig. 3a). The three areas of coexistence are still present and inherit the same mechanisms as in the system without diffusion. The middle area of coexistence (yellow area with black hatching) occurs because of spatial segregation, where each species achieves a biomass maximum in a different patch (Fig. S3, point $i i$ ). In this case, the two side areas of coexistence occur due to resource partitioning in one or another patch, when the maximum of biomass of both species is located in the same patch (Fig. S3, point $i$ ). A further increase of the diffusion rate decreases the difference in resource concentrations between patches, shrinking the regional coexistence range (hatched yellow area). At the same time, the ranges of local coexistence (yellow area without hatching in Fig. 3a-d) increase in size and their boundaries approach each other. Additionally, the M-monoculture areas become larger and shift closer to the diagonal of the resource plane (as indicated by arrows in Fig. $3 b, c)$. Increasing the diffusion rate further eventually leads to an overlap of the M-monoculture areas, resulting in a regime of bistability, where either species 1 or species 2 can win in the entire system in dependence on the initial conditions (Fig. 3d, white). When species 2 wins, it achieves a biomass maximum in patch $A$ and when species 1 wins, it has a biomass maximum in patch B (Fig. S3, point $i v$ ).

At an even higher diffusion rate, the M-monoculture areas shift further and overlap with the areas of local coexistence, leading to another kind of bistability, when either one species wins or both species coexist (light blue and light red shading in Fig. 3e-g). At the same time, the areas of local coexistence also start to overlap, leading to a third bistability regime characterized by two alternative coexistence regimes (yellow dotted area in Fig. 3f, g). In this third bistability regime, both species coexist, but one or the other species achieves a larger biomass depending on initial conditions (Fig. S3, point vi). Finally, at very high diffusion rates, we obtain a pattern that is expected for homogeneous systems (Fig. 3h), where the Mmonoculture areas disappear as both patches become equal in terms of resource conditions, and the bistable coexistence regimes merge into one stable state of coexistence at balanced resource ratios.

To study details of the transitions in species composition, which include a surprisingly large variety of alternative stable states, consider the evolution of the bifurcation diagram of competition outcomes with increasing diffusion. We characterize the species composition by the relative biomass of species 2 with respect to the total biomass, $n_{2}=N_{2} /\left(N_{1}+N_{2}\right)$. This value changes from 0 if only species 1 is present to 1 if species 2 wins the competition. Figure 4 shows $n_{2}$ calculated along a gradient of resource supply ratios (along the dashed diagonal line in Fig. 2a) for the same diffusion rates and heterogeneity level as in Fig. 3. To find the bistable regimes, we show results obtained for different initial conditions, with blue markers when species 1 is the resident and species 2 invades, or with red markers for the opposite sequence of species introduction.

In a nearly homogeneous system with a large diffusion rate (Fig. $4 \mathrm{~h}$ ), $n_{2}$ follows a sigmoidal pattern: it equals 0 , when $S_{2} /$ $S_{1}$ is small and only a good competitor for resource 1 (species 

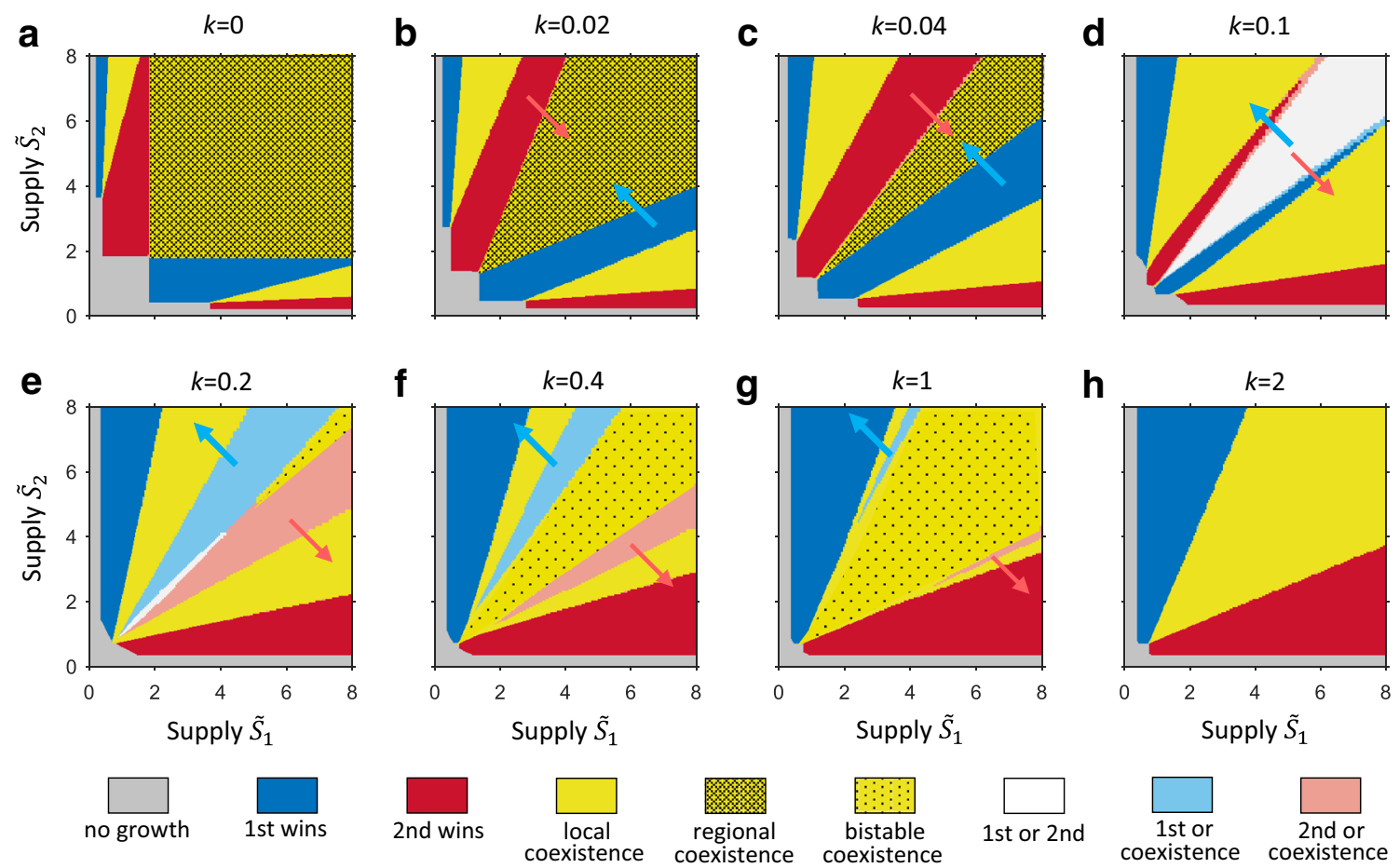

Fig. 3 Effect of diffusion on competition outcomes under strongly heterogenous distribution of supplied resources. $\mathbf{a}-\mathbf{h}$ show competition outcomes in a gradient from strongly heterogenous conditions when diffusion rate $k=0$ ( $\mathbf{a}$ is the same as Fig. $2 b$ ) to the conditions when the

resource and biomass distributions become homogenized due to a large diffusion rate (h approaches Fig. 2a). The red and blue arrows indicate the shift of the M-monoculture areas with increasing diffusion rate

1) survives; $n_{2}$ increases in the intermediate range of supply ratios, where both species coexist and the dominance gradually shifts from species 1 to species 2 ; and it equals 1 for large $S_{2} / S_{1}$ where species 2 (a good competitor for resource 2) wins. By contrast, in the limit of small diffusion (Fig. 4a), the competition outcome consists of two such sigmoidal patters,

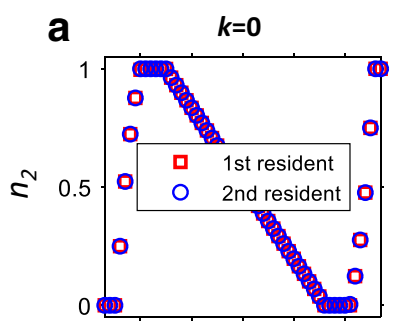

b
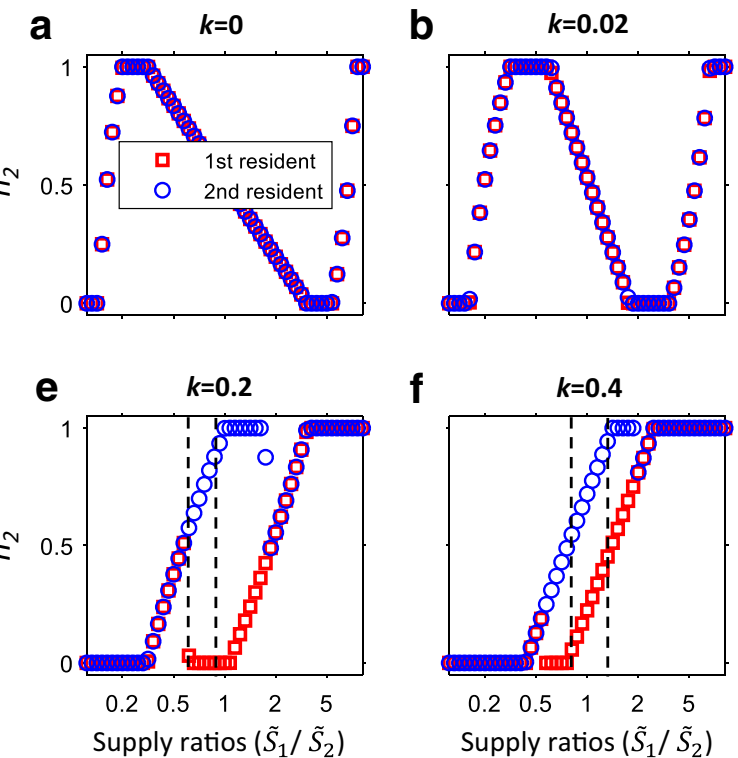

f

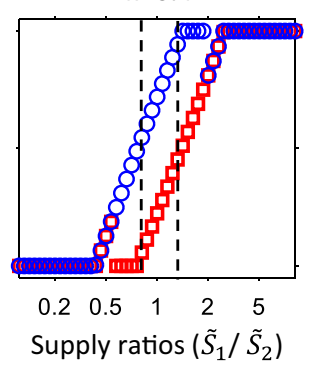

Fig. 4 Bifurcation analysis of the competition outcomes shown in Fig. 3. The relative biomass of species $2, n_{2}=N_{2} /\left(N_{1}+N_{2}\right)$, is calculated along the gradient of resource supply ratios (black dashed line in Fig. 2a). To

where $n_{2}$ increases with $S_{2} / S_{1}$, and an intermediate part, where $n_{2}$ decreases with $S_{2} / S_{1}$. The sigmoidal parts correspond to the transition in species composition when the competitors coexist locally within one patch. The intermediate part manifests a transition of the biomass maximum from patch $\mathrm{A}$ to patch $\mathrm{B}$, when the species coexist regionally (see also Fig. 2e, f).
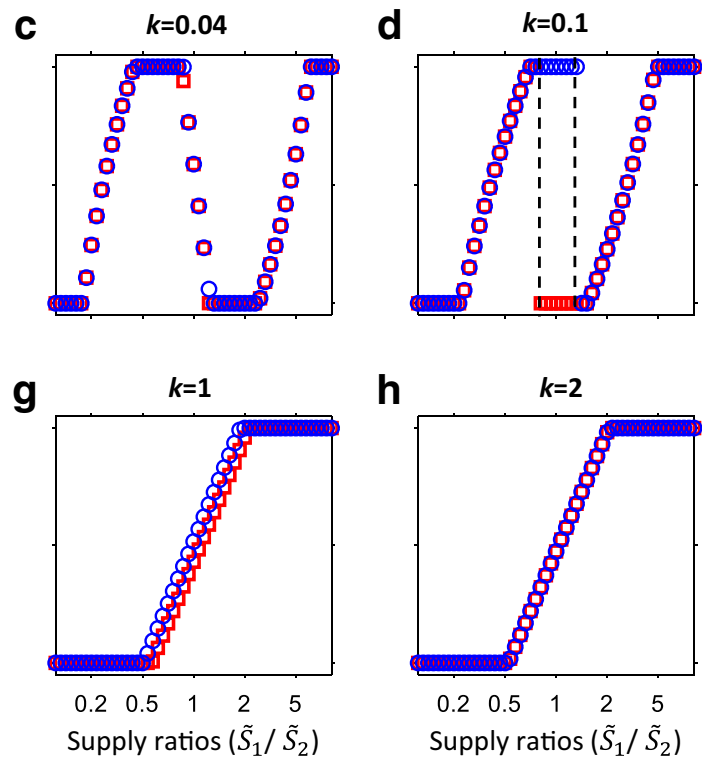

detect bistable outcomes, we simulate different invasion sequences: when species 1 is the resident and species 2 invades (red squares) and the opposite sequence (blue circles) 
If the diffusion rate is greater than zero, the biomass of competitors can be present in both patches and the difference between the regional and local coexistence is not so evident. We still can distinguish them using the property of $n_{2}$ to increase with $S_{2} / S_{1}$, when species coexist due to local resource partitioning and to decrease with $S_{2} / S_{1}$ when the coexistence is regional. The first property occurs because an increase of $S_{2} /$ $S_{1}$ relaxes the local competition for resource 2 , which should favor species 2 and, therefore, increase $n_{2}$. However, a decrease of $n_{2}$ with $S_{2} / S_{1}$ means that the local resource partitioning is not the main mechanism providing species coexistence and therefore species coexist mainly due to spatial segregation.

With increasing diffusion rate, the two sigmoidal patterns expand and the intermediate range shrinks (Fig. 4a-c). This transformation reflects the shrinking of the regional coexistence range and the widening of local coexistence ranges with diffusion, shown in Fig. 3a-c. At diffusion rate $k=0.1$, the two sigmoidal patterns begin to overlap and the regional coexistence at intermediate resource ratios is replaced by bistability between the M-monoculture of either one or another competitor (Fig. 4d). A further increase of diffusion leads to a larger overlap of the two sigmoidal parts, resulting additionally either in bistability between a M-monoculture and species coexistence (Fig. 4e) or in bistability between two stable coexistence states (Fig. 4f, g). Finally, the two sigmoidal patterns merge together, the bistable regimes converge to an intermediate state, and we obtain one sigmoidal pattern which describes the influence of the resource ratio on species composition in a homogeneous system (Fig. 4h).

Such strong effects of the diffusion rate on species composition are manifested only under extremely heterogeneous conditions, and they gradually disappear with decreasing resource heterogeneity. Figure 5 summarizes the overall effects of diffusion rate on the patterns of competition outcomes for small (left), intermediate (middle), and high (right) levels of resource heterogeneity. In contrast to the effects of the diffusion rate, a decrease of resource heterogeneity does not turn the sectors of different competition outcomes over the resource plane. Instead, the M-monocultures and bistability ranges decrease in size (Fig. 5 middle and right panel) and finally disappear, so that the patterns of competition outcomes approach those for homogeneous systems and become independent of the diffusion rate (Fig. 5, left column).

The size of the area where all species become extinct (gray) depends also strongly on the diffusion rate and resource heterogeneity. At a low heterogeneity level $a$, this area has the smallest size and becomes nearly independent of the diffusion rate, as its boundary is defined by the species $R^{*}$-values. This area increases when the system becomes more heterogeneous, and it achieves a maximal size in a strongly heterogeneous system with a small diffusion rate (Fig. 51). Under these conditions, the species effective minimal resource requirements reach maximal values (Fig. $\mathrm{S} 4 \mathrm{~A}$ ) and the species total biomass drops to its minimum (Fig. S4B).

Finally, we analyze the effects of diffusion and resource heterogeneity on regional and local biodiversity. First, we focus on the aggregated biodiversity, averaged over a gradient of resource ratios located along the dashed diagonal line in the resource plane (Fig. 2a). Regional average diversity reaches a maximum at zero diffusion and monotonically decreases with increasing diffusion (Fig. 6a), while local average diversity has a unimodal dependence on the diffusion rate with a maximum at an intermediate diffusion rate (Fig. 6b). These patterns hold when the system is heterogeneous in resource distributions ( $a>0.65$, Fig. 6), while at low resource heterogeneity, the between-patch resource gradients are weak and the effects of diffusion disappear. Thus, under heterogeneous conditions, both the regional and local aggregated biodiversity follow the intuitively expected dependencies, as suggested by Mouquet and Loreau (2003). Furthermore, both local and regional diversity show a unimodal dependence on resource heterogeneity, when diffusion is relatively weak $(k<0.08$, Fig. 6).

However, the diversity patterns averaged over a range of resource ratios only partly reflect the patterns obtained for specific ratios of resource supplies. For an imbalanced resource supply ratio $\left(S_{1} / S_{2}=0.33\right)$, regional and local diversity reach a maximum at high levels of resource heterogeneity and low diffusion rates (Fig. 7, left panel), as these conditions favor species coexistence at imbalanced resource ratios (Fig. 5). Local diversity also drops at low diffusion due to the absence of source-sink dynamics. At a relatively balanced resource supply ratio $\left(S_{1} / S_{2}=0.66\right)$, two main changes are observed, which hold for both regional and local diversity. First, high diversity values are obtained for a wider range of heterogeneity and diffusion levels (Fig. 7, middle panel), as the possibility of regional and local coexistence increases at a more balanced resource supply ratio (Fig. 5). Second, a diversity decline appears at high $a$ and intermediate $k$ levels. This drop occurs because at these conditions, the system becomes bistable and only one of the competitors survives (Fig. 5). Finally, at a balanced resource supply ratio $\left(S_{1} / S_{2}=1\right)$, regional and local diversity reach the maximum value for most levels of resource heterogeneity and diffusion rates (Fig. 7, right panel), as the competitors can coexist due to local resource partitioning. However, the bistability in the competition outcomes leads to a reduction of regional and local diversity at high $a$ and intermediate $k$.

\section{Discussion}

Understanding the mechanisms shaping species composition and diversity in heterogeneous environments can improve predictions on ecosystem structure and functioning (Tilman et al. 
Fig. 5 Effects of diffusion rate and resource heterogeneity on the patterns of competition outcomes. Different competition outcomes are color coded (see legend)
Fig. 6 Effects of diffusion and resource heterogeneity on averaged regional (a) and local (b) diversity. Diversity is measured as the effective species number (exponential Shannon index) and was averaged along the gradient of resource supply ratios (black dashed line in Fig. 3h)

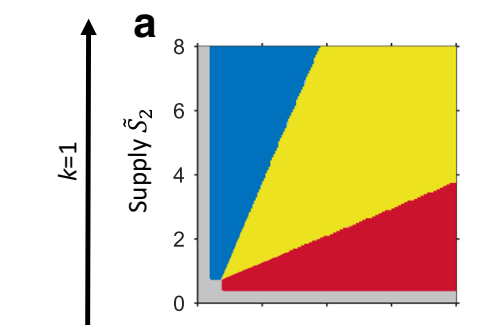

b
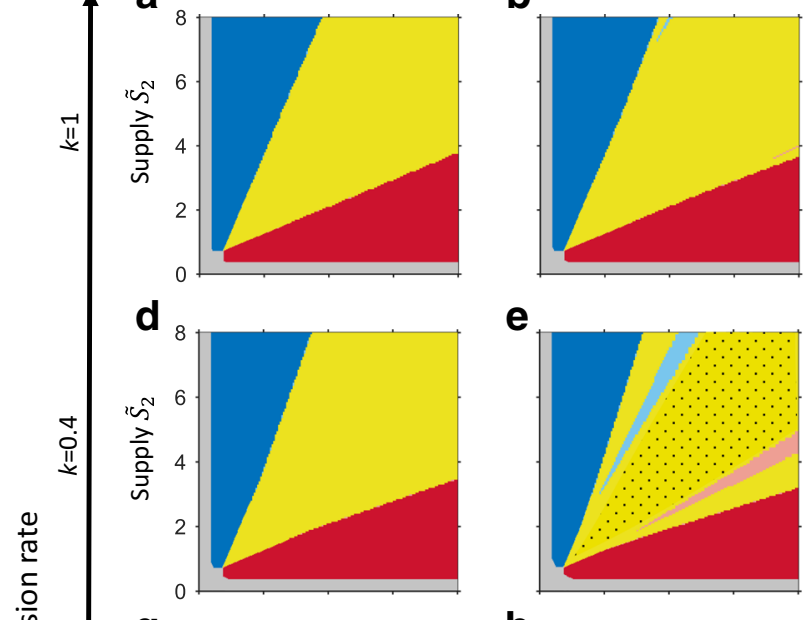

e

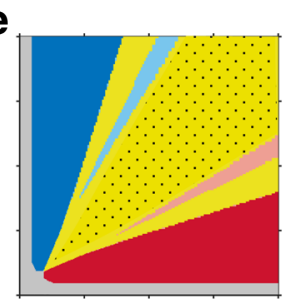

h

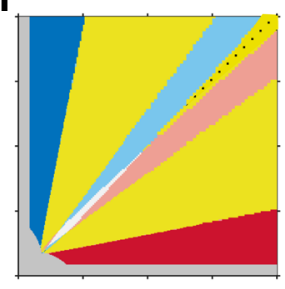

k

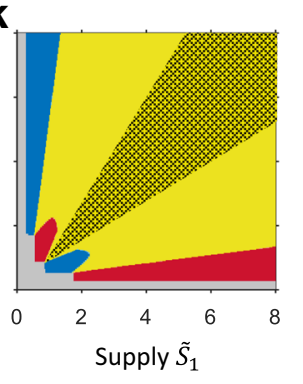

C

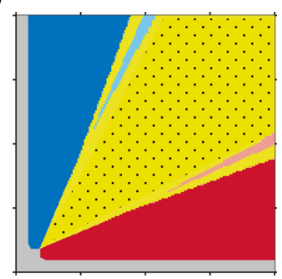

f

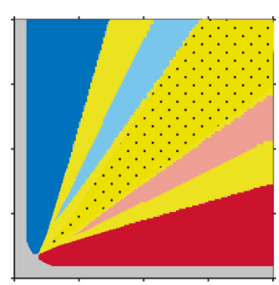

i

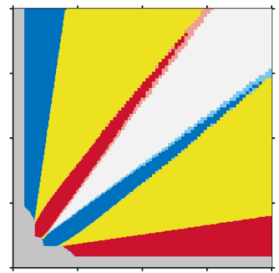

I

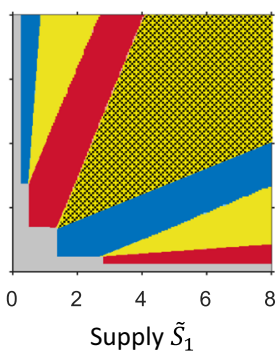

$a=0.8$

$a=0.9$

Resource heterogeneity
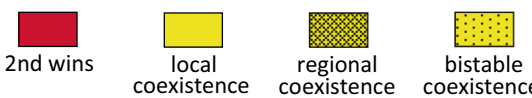

1st or 2nd

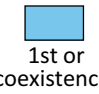

2nd or coexistence b

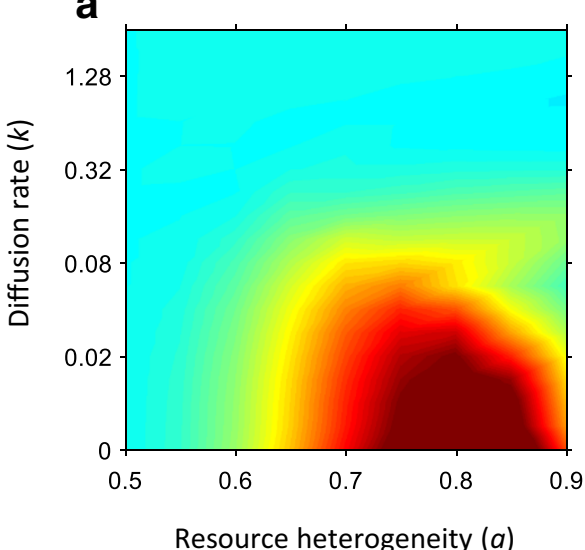

Local diversity

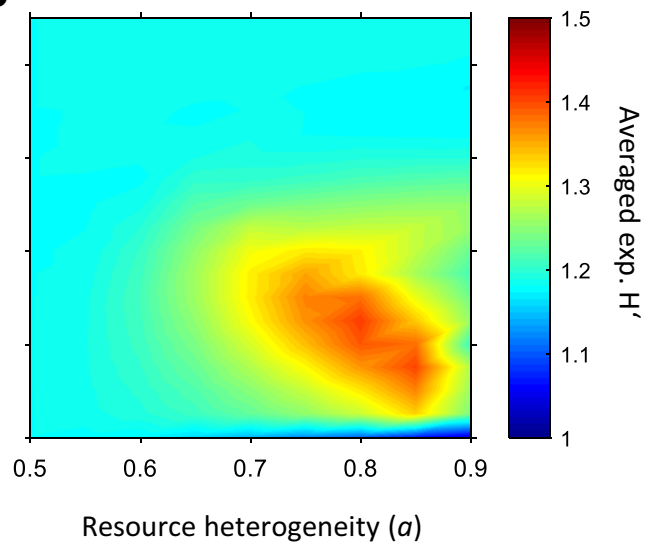



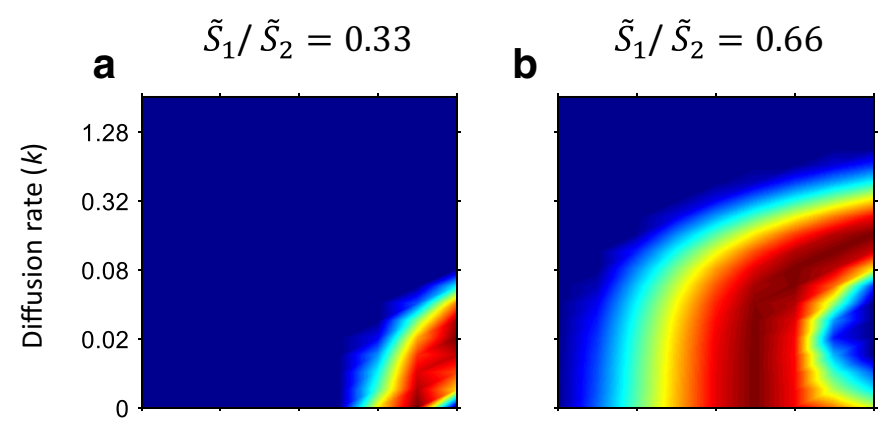

c $\quad \tilde{S}_{1} / \tilde{S}_{2}=1$
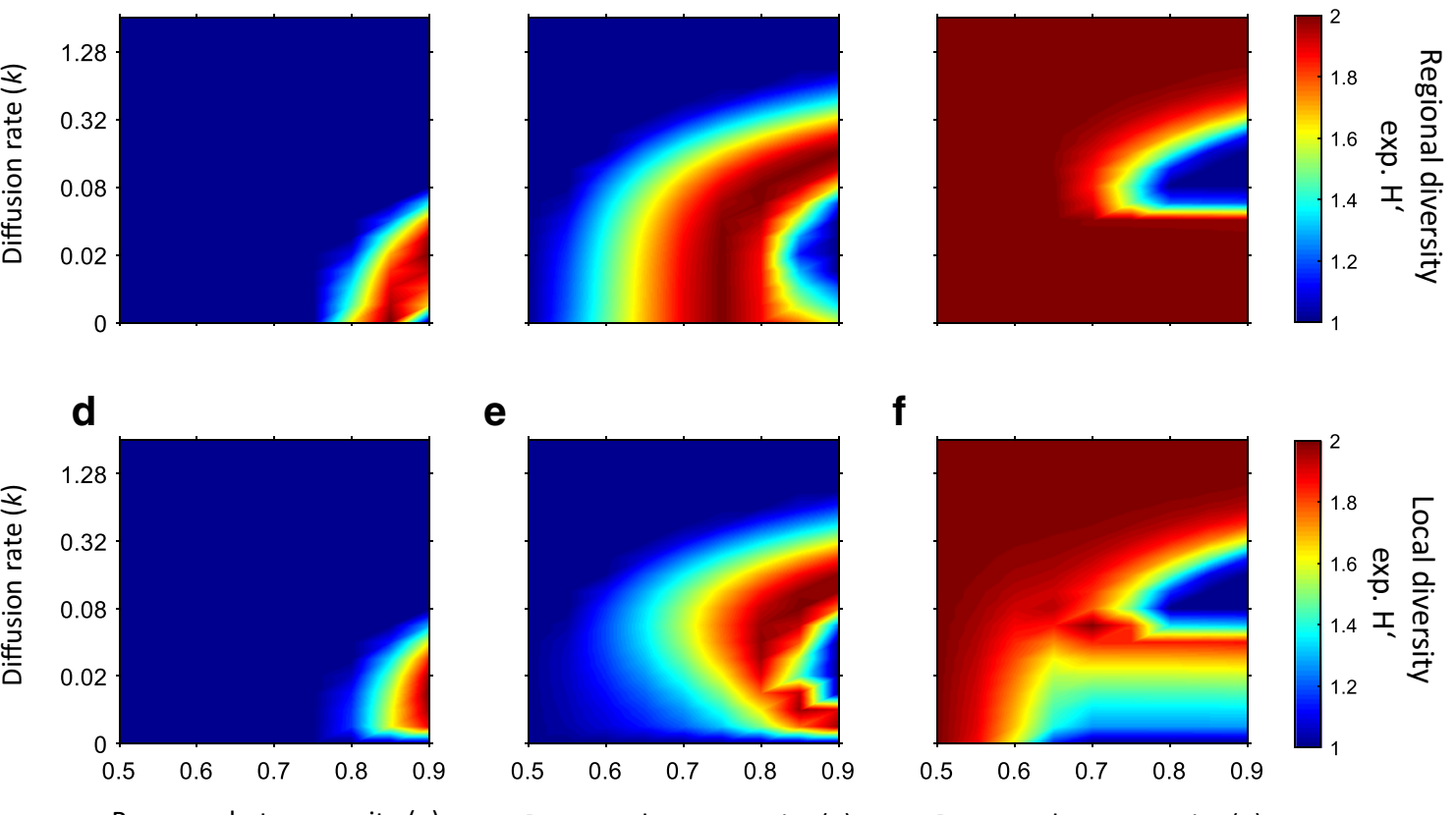

e

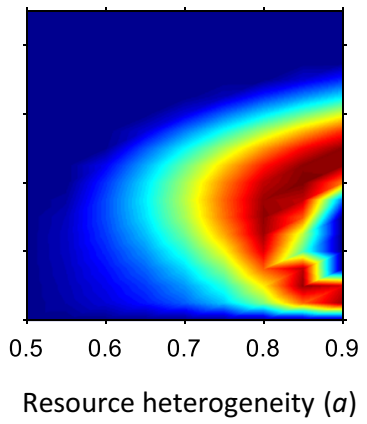

f

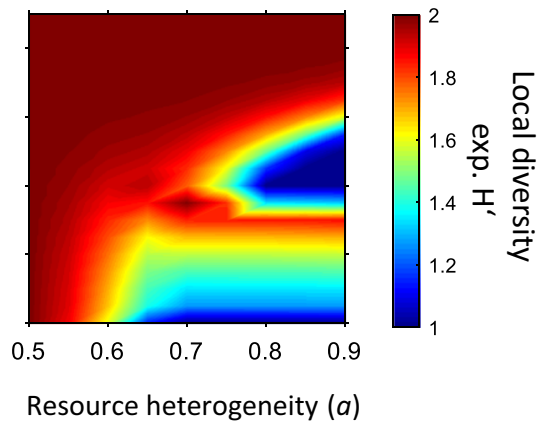

Fig. 7 Effects of diffusion and resource heterogeneity on regional (a) and local diversity (b) for various resource supply ratios. The same as Fig. 6, but the results are not averaged along the resource ratio gradient and

2014). Here, we model resource competition of two species for two resources in a two-patch metaecosystem, to understand the effects of resource heterogeneity, resource stoichiometry, and environmental connectivity on species coexistence and diversity. We generalized the gradostat experimental setup suggested by Lovitt and Wimpenny (1981)), in order to control independently the level of resource heterogeneity, stoichiometry, and the absolute level of supplied resources. Regulating the level of the system's heterogeneity, we illustrate the emergent properties for different metaecosystem scenarios and compare them with outcomes obtained for a homogeneous system.

\section{Patterns of competition outcomes}

When resource distribution is uniform, in accordance with resource ratio theory, we obtain local coexistence of species for balanced resource supply ratios and dominance of one of the competitors at imbalanced resource ratios (Tilman 1980, and Fig. $2 \mathrm{a}$ in this paper). The presence of asymmetry in the resource supply distribution between the patches changes significantly the pattern of competition outcomes (Fig. 2b). Previous theoretical work (Jäger et al. 1987, Smith and Waltman 1991) and recent research (Ryabov et al. 2010; Ryabov and Blasius 2011; Haegeman and Loreau 2015) have established the fact that regional coexistence is a dominant outcome in heterogeneous environments. Our analysis offers plotted separately for imbalanced (left), relatively balanced (middle) and balanced (right) ratios of resource supplies (See the location of the three resource supply ratios in Fig. S1)

a more detailed view on the patterns of species coexistence under different metaecosystem scenarios.

When the resource distribution is strongly heterogeneous, we obtain three areas of species coexistence in the resource supply plane (Fig. 2b). The middle of the three coexistence areas manifests regional species coexistence, which occurs due to spatial segregation, or spatial storage effect (Chesson 2000). This type of coexistence occurs at balanced total resource supply ratios because the asymmetry in resource allocation leads to different local resource ratios in each patch, and each competitor obtains a niche in the patch that better matches its traits. The other two coexistence areas appearing at imbalanced supply ratios are attributed to local coexistence. This occurs because the asymmetry of resource distribution compensates the imbalance in resource supplies in one of the patches, where the local resource ratios become suitable for local coexistence due to resource partitioning (Fig. 2e, f).

The monoculture outcomes also follow a pattern strikingly different from that obtained for uniform systems. For instance, an increase of the resource supply ratio $S_{2} / S_{1}$ from a balanced ratio in a uniform system causes a transition from coexistence to the dominance of species 1 (a good competitor for $R_{1}$ ). On the other hand, if resource heterogeneity is large, an increase in $S_{2} / S_{1}$ first causes a transition from regional coexistence to a monoculture of species 2 , and only then, through the local coexistence regime, to a monoculture of species 1 . The first regime (called M-monoculture here) occurs because concentrations of $R_{1}$ in patch $\mathrm{B}$ become too low for both species, but 
patch $\mathrm{A}$, due to the asymmetry in resource allocation, still receives more of $R_{1}$ than $R_{2}$ and species 2 wins. The second regime (termed here as A-monoculture) occurs when extremely large $S_{2} / S_{1}$ ratios override the effects of the resource allocation asymmetry, both patches become $R_{1}$-limited, and species 1 wins. Thus, the M-monoculture regime is shaped by the interplay of the asymmetry in resource allocation and resource supply ratio, and the A-monoculture regime has the same mechanism as in well-mixed systems.

\section{Alternative stable states}

Alternative stables states have been described in theoretical models of species competition on spatial gradients (Ryabov and Blasius 2011, 2014). Shurin et al. (2004) have shown that competitors exhibiting alternative stable states when initial heterogeneity is biotically generated can nevertheless coexist if the spatial heterogeneity is maintained abiotically (Shurin et al. 2004). In a series of previous works, Ryabov and Blasius $(2011,2014)$ have shown that the effect can be also reversed. Namely, in the presence of inverse resource gradients, species which can coexist in a uniform system due to resource partitioning can biotically (via resource consumption) generate bistable states and vice versa. Here, we extend these results and show that inverse gradients of two resources can support a variety of bistable states: between the dominance of one or another competitor, between a single species dominance and coexistence, and bistable coexistence regimes.

Why does the same combination of consumption coefficients favor species coexistence in uniform systems but leads to bistable outcomes in the presence of inverse resource gradients? To answer this question, consider the different mechanisms of coexistence in homogeneous and heterogeneous systems. In homogeneous systems, coexistence occurs when species have a trade-off in resource requirements and each species consumes relatively more its most (in relative terms) required resource. Then, at balanced resource inputs, the population of each species becomes self-limited by its most required resource, leaving a free niche for its competitor. In contrast, in the presence of inverse resource gradients, coexistence can occur due to species sorting, when each species occupies a most suitable patch. This mechanism requires only a trade-off in resource requirements, but not a special combination of consumption coefficients. The consumption coefficients in such systems drive, in particular, the strength of mass effects occurring due to biomass diffusion from favorable to unfavorable patches.

For instance, in the gradostat setup shown in Fig. 1 (when $\alpha=1$ ), patch $A$ is favorable and patch $B$ is unfavorable for species 2 , as this species has relatively high requirements for resource $R_{1}$. If species 2 consumes predominantly resource $R_{1}$, the mass effect in patch B can become strong, such that the depletion of $R_{1}$ in patch $\mathrm{B}$ can even prohibit growth of species
1 , despite its lower requirements for $R_{1}$. In the same way, species 1 when present can also suppress the growth of species 2 , and we can find that a combination of consumption coefficients, leading to coexistence in uniform systems, can favor alternative stable states in the presence of inverse resource gradients.

Furthermore, it becomes clear that coexistence in a heterogeneous system is possible if each species consumes relatively less of its most required resource, because this reduces the mass effect of each species in their unfavorable patches, leading to smaller resource gradients. Our model confirms this conjecture (not presented here) and shows that similar to this effect in continuous systems (Ryabov and Blasius 2011, 2014), parameters leading to bistability in uniform systems can lead to coexistence in a wide range of resource supply ratios in a patchy environment.

These bistable equilibria require a strong feedback of biomass fluxes on resource gradients, which causes an effect of the initial biomass distribution on the final distribution of resources and species composition (Ryabov et al. 2010; Ryabov and Blasius 2011). Analysis of the separate effects of species and resource diffusion (will be presented elsewhere) indicates that the main driver of these kinds of bistability is species dispersal. This is because species dispersal causes strong mass effect which increases the steepness of resource gradients. In this way, for instance, diffusion of a resident population into an unfavorable patch can deplete resource concentrations there to a level at which an invader population cannot survive. Consequently, the appearance of such bistable regimes requires strong levels of resource heterogeneity, large biomass maintained by large resource inputs, and intermediate levels of between-patch diffusion. Natural examples, at which such alternative stable states may appear, can be found in spatial systems with strong nutrient gradients, e.g., coastal to open ocean waters which typically differ in nitrogen to phosphorus ratios of resource supplies (Arrigo 2005; Moore et al. 2013).

\section{Resource use efficiency and limiting requirements}

Our model predicts that resource heterogeneity has a negative effect on the total biomass and resource use efficiency, supporting the notion that competitive interactions affect ecosystem functioning (Gross and Cardinale 2007; Hillebrand and Matthiessen 2009). In the resource plane, the area where all species become extinct reaches its maximum at the largest levels of resource heterogeneity and lowest diffusion rates. Under strongly heterogeneous conditions, the patch-local concentrations of one resource can fall below species $\mathrm{R}^{*}$ values, while concentrations of the other resource in this patch can strongly exceed this minimal level. This leads to a large local resource surplus which is associated with the increase of effective minimal resource requirements (community $\mathrm{R}^{*}$ ) and the decline of total biomass. These results are in agreement 
with theoretical predictions of meta-community models (Abrams and Wilson 2004; Gross and Cardinale 2007), metaecosystem models (Codeço et al. 2001; Martines et al. 2013; Marleau et al. 2015), and experimental observations (Codeço et al. 2001; Gülzow et al. 2018) .

\section{Biodiversity patterns}

Our modeling approach has many parallels with the model suggested by Mouquet et al. (2006), who studied the influence of species dispersal in a two-patch metacommunity. Here, we make a step further, including the influence of resource fluxes and stoichiometry of resource supplies, adding therefore two more dimensions to the space of parameters. Meta-community theory has highlighted the role of source-sink dynamics on biodiversity, predicting a decreasing regional diversity with increasing dispersal and a maximum of local diversity at intermediate levels of dispersal (Mouquet and Loreau 2003; Cadotte 2006), with both regional and local diversity achieving their maximum at intermediate heterogeneity levels (Mouquet et al. 2006). However, these predictions may not be universal under metaecosystem conditions where resource flows play an additional role (Haegeman and Loreau 2014).

We show in our study that patterns of regional and local diversity averaged over a gradient of resource ratios agree with previous findings: regional diversity declines monotonically with between-patch diffusion, local diversity peaks at intermediate levels of diffusion, and both local and regional diversities achieve a maximum at intermediate levels of resource heterogeneity (Fig. 6). However, if results are not averaged along resource gradients, they support the generality of previous findings only partly, as the model demonstrates different patterns for balanced and imbalanced resource inputs. For instance, for imbalanced total resource supplies (Fig. 7, left panel), regional and local diversities have similar dependences on diffusion as for the aggregated patterns, but unlike (Mouquet et al. 2006), both diversity measures reach their maximum at the highest level of resource heterogeneity, because under these conditions, the imbalance in resource input can be compensated in one of the patches by the asymmetry of resource distribution, leading to local coexistence in this patch. By contrast, when resource inputs are balanced, the system can exhibit a variety of alternative stable states which can reduce biodiversity at intermediate dispersal rates. As a result, at high heterogeneity levels, both local and regional diversity follow a bimodal (or multimodal) dependence on diffusion with a drop at intermediate diffusion rates (Fig. 7, middle and right panel). These patterns also correlate with results by Haegeman and Loreau (2014) who showed that, depending on the interaction between species dispersal and resource flows, the local diversity can be either unimodal, monotonically increasing, or multimodal.

\section{Concluding remarks}

Gradients in resource distributions take place over regional scales within ecosystems (Herbert et al. 2004; Riesch et al. 2018) and between ecosystems (Baxter et al. 2004; Gratton and Zanden 2009). Understanding the mechanisms driving species composition and diversity in heterogeneous environments is crucial for ecosystem management (Pickett and Cadenasso 1995; Loreau et al. 2003a). Our proposed model combines modern metaecosystem dynamics and classic mechanisms of resource competition, offering prediction on species composition and biodiversity in heterogeneous environments. We focus on resource competition for two limiting resources, analyzing the patterns of regional and local diversity at different metaecosystem scenarios, and highlighting the interactive effects of resource heterogeneity and diffusion on them. The model predictions and methodology can be used to understand ecosystem structure and functioning in large-scale ecosystem models, local scale simulations, and experimental studies.

Acknowledgments I.T. acknowledges support from Deutsche Forschungsgemeinschaft (DFG), project GZ: RY 143/1-1. ABR acknowledges the Lower Saxony Ministry for Science and Culture, project POSER.

\section{References}

Abrams PA, Wilson WG (2004) Coexistence of competitors in metacommunities due to spatial variation in resource growth rates; does $\mathrm{R}^{*}$ predict the outcome of competition? Ecol Lett 7:929-940. https://doi.org/10.1111/j.1461-0248.2004.00644.x

Arrigo KR (2005) Marine microorganisms and global nutrient cycles. Nature 437:349-355. https://doi.org/10.1038/nature04159

Baxter CV, Fausch KD, Murakami M, Chapman PL (2004) Fish invasion restructures stream and forest food webs by interrupting reciprocal prey subsidies. Ecology 85:2656-2663. https://doi.org/10.1890/04138

Cadotte MW (2006) Dispersal and species diversity: a meta-analysis. Am Nat 167:913-924

Chesson P (2000) Mechanisms of maintenance of species diversity. Annu Rev Ecol Syst 31:343-366

Codeço CT, Grover JP, DeAngelis AEDL (2001) Competition along a spatial gradient of resource supply: a microbial experimental model. Am Nat 157:300-315. https://doi.org/10.1086/319195

Gounand I, Mouquet N, Canard E et al (2014) The paradox of enrichment in metaecosystems. Am Nat 184:752-763. https://doi.org/10.1086/ 678406

Gounand I, Harvey E, Ganesanandamoorthy P, Altermatt F (2017) Subsidies mediate interactions between communities across space. Oikos 126:972-979. https://doi.org/10.1111/oik.03922

Gratton C, Zanden MJV (2009) Flux of aquatic insect productivity to land: comparison of lentic and lotic ecosystems. Ecology 90: 2689-2699. https://doi.org/10.1890/08-1546.1

Gravel D, Guichard F, Loreau M, Mouquet N (2010a) Source and sink dynamics in meta-ecosystems. Ecology 91:2172-2184. https://doi. org/10.1890/09-0843.1

Gravel D, Mouquet N, Loreau M, Guichard F (2010b) Patch dynamics, persistence, and species coexistence in metaecosystems. Am Nat 176:289-302. https://doi.org/10.1086/655426 
Gravel D, Massol F, Leibold MA (2016) Stability and complexity in model meta-ecosystems. Nat Commun 7:12457-12458. https://doi. org $/ 10.1038 /$ ncomms 12457

Gross K, Cardinale BJ (2007) Does species richness drive community production or vice versa? Reconciling historical and contemporary paradigms in competitive communities. Am Nat 170:207-220. https://doi.org/10.1086/518950

Grover JP (1997) Resource Competition. https://link.springer.com/book/ 10.1007\%2F978-1-4615-6397-6. Accessed 26 Aug 2017

Gülzow N, Wahlen Y, Hillebrand H (2018) Metaecosystem dynamics of marine phytoplankton alters resource use efficiency along stoichiometric gradients. Am Nat:000-000. https://doi.org/10.1086/700835

Haegeman B, Loreau M (2014) General relationships between consumer dispersal, resource dispersal and metacommunity diversity. Ecol Lett 17:175-184. https://doi.org/10.1111/ele.12214

Haegeman B, Loreau M (2015) A graphical-mechanistic approach to spatial resource competition. Am Nat 185:E1-E13. https://doi.org/ 10.1086/679066

Herbert DA, Rastetter EB, Gough L, Shaver GR (2004) Species diversity across nutrient gradients: an analysis of resource competition in model ecosystems. Ecosystems 7:296-310. https://doi.org/10. 1007/s 10021-003-0233-x

Hillebrand H, Matthiessen B (2009) Biodiversity in a complex world: consolidation and progress in functional biodiversity research. Ecol Lett 12:1405-1419. https://doi.org/10.1111/j.1461-0248. 2009.01388.x

Hodapp D, Hillebrand H, Blasius B, Ryabov AB (2016) Environmental and trait variability constrain community structure and the biodiversity-productivity relationship. Ecology 97:1463-1474. https://doi.org/10.1890/15-0730.1

Huisman J, Weissing FJ (1994) Light-limited growth and competition for light in well-mixed aquatic environments: an elementary model. Ecology 75:507-520

Leibold MA, Holyoak M, Mouquet N et al (2004) The metacommunity concept: a framework for multi-scale community ecology. Ecol Lett 7:601-613. https://doi.org/10.1111/j.1461-0248.2004.00608.x

Leinster T, Cobbold CA (2012) Measuring diversity: the importance of species similarity. Ecology 93:477-489. https://doi.org/10.1890/102402.1

León JA, Tumpson DB (1975) Competition between two species for two complementary or substitutable resources. J Theor Biol 50:185-201. https://doi.org/10.1016/0022-5193(75)90032-6

Limberger R, Birtel J, Farias DDS, Matthews B (2017) Ecosystem flux and biotic modification as drivers of metaecosystem dynamics. Ecology 98:1082-1092. https://doi.org/10.1002/ecy.1742

Loreau M, Mouquet N, Gonzalez A (2003a) Biodiversity as spatial insurance in heterogeneous landscapes. Proc Natl Acad Sci 100: 12765-12770. https://doi.org/10.1073/pnas.2235465100

Loreau M, Mouquet N, Holt RD (2003b) Meta-ecosystems: a theoretical framework for a spatial ecosystem ecology. Ecol Lett 6:673-679. https://doi.org/10.1046/j.1461-0248.2003.00483.x

Lovitt RW, Wimpenny JWT (1981) The gradostat: a bidirectional compound chemostat and its application in microbiological research. Microbiology 127:261
Marleau JN, Guichard F, Loreau M (2015) Emergence of nutrient colimitation through movement in stoichiometric meta-ecosystems. Ecol Lett 18:1163-1173. https://doi.org/10.1111/ele.12495

Martines IP, Kojouharov HV, Grover JP (2013) Nutrient recycling and allelopathy in a gradostat. Comput Math Appl 66:1613-1626. https://doi.org/10.1016/j.camwa.2013.02.005

Moore CM, Mills MM, Arrigo KR et al (2013) Processes and patterns of oceanic nutrient limitation. Nat Geosci 6:701-710. https://doi.org/ 10.1038/ngeo1765

Mouquet N, Loreau M (2003) Community patterns in source-sink metacommunities. Am Nat 162:544-557. https://doi.org/10.1086/ 378857

Mouquet N, Moore JL, Loreau M (2002) Plant species richness and community productivity: why the mechanism that promotes coexistence matters. Ecol Lett 5:56-65. https://doi.org/10.1046/j.14610248.2002.00281.x

Mouquet N, Miller TE, Daufresne T, Kneitel JM (2006) Consequences of varying regional heterogeneity in source-sink metacommunities. Oikos 113:481-488. https://doi.org/10.1111/j.2006.0030-1299. 14582. $\mathrm{x}$

Pickett STA, Cadenasso ML (1995) Landscape ecology: spatial heterogeneity in ecological systems. Science 269:331-334. https://doi.org/ 10.1126/science.269.5222.331

Riesch R, Plath M, Bierbach D (2018) Ecology and evolution along environmental gradients. Curr Zool 64:193-196. https://doi.org/10. 1093/cz/zoy015

Ryabov AB, Blasius B (2011) A graphical theory of competition on spatial resource gradients. Ecol Lett 14:220-228. https://doi.org/ 10.1111/j.1461-0248.2010.01574.x

Ryabov AB, Blasius B (2014) Depth of the biomass maximum affects the rules of resource competition in a water column. Am Nat 184:E132E146. https://doi.org/10.1086/677544

Ryabov AB, Rudolf L, Blasius B (2010) Vertical distribution and composition of phytoplankton under the influence of an upper mixed layer. J Theor Biol 263:120-133. https://doi.org/10.1016/j.jtbi.2009. 10.034

Shurin JB, Amarasekare P, Chase JM et al (2004) Alternative stable states and regional community structure. J Theor Biol 227:359-368

Tilman D (1980) Resources: a graphical-mechanistic approach to competition and predation. Am Nat 116:362-393. https://doi.org/10. $2307 / 2463311$

Tilman D (1982) Resource competition and community structure. Princeton University Press

Tilman D (1985) The resource-ratio hypothesis of plant succession. Am Nat 125:827-852

Tilman D, Isbell F, Cowles JM (2014) Biodiversity and ecosystem functioning. Annu Rev Ecol Evol Syst 45:471-493. https://doi.org/10. 1146/annurev-ecolsys-120213-091917

Wilson DS (1992) Complex interactions in metacommunities, with implications for biodiversity and higher levels of selection. Ecology 73:1984-2000. https://doi.org/10.2307/1941449 